\title{
Comparing bivalent and quadrivalent HPV vaccines
}

In the third paragraph from the end in this editorial by René $\mathrm{H}$ M Verheijen (BMJ 2011;343:d5720, doi:10.1136/bmj.d5720) the author mistakenly referred to deaths instead of cases. The fourth sentence should have read: "It has been estimated that the Italian programme, which uses the bivalent vaccine, would prevent 295 more cases of cancer [not " 295 more deaths from cancer"] but 25848 fewer cases of genital warts than if it used the quadrivalent vaccine."

Cite this as: BMJ 2011;343:d6786

๑ BMJ Publishing Group Ltd 2011 